\title{
Physical specifications of photonic crystal slab lenses and their effects on image quality
}

\author{
Sohrab Safavi,,** Rahim Ghayour, ${ }^{2}$ and Jonas Ekman ${ }^{1}$ \\ ${ }^{1}$ Department of Computer Science, Electrical \& Space Engineering, Luleå University of Technology, Luleå, Sweden \\ ${ }^{2}$ Department of Electronic and Communications, Electrical Engineering School, Shiraz University, Shiraz, Iran \\ ${ }^{*}$ Corresponding author: Sohrab.Safavi@LTU.SE
}

Received February 13, 2012; revised May 29, 2012; accepted May 29, 2012; posted May 30, 2012 (Doc. ID 163004); published June 28, 2012

\begin{abstract}
Photonic crystal $(\mathrm{PhC})$ lenses with negative refractive index have attracted intense interest because of their application in optical frequencies. In this paper, two-dimensional $\mathrm{PhC}$ lenses with a triangular lattice of cylindrical holes in dielectric material are investigated. Various physical parameters of the lens are introduced, and their effects on the lens response are studied in detail. The effect of the surface termination is investigated by analyzing the power flux within the PhC structure. A new lens formula has been obtained that shows a linear relation between the source distance (distance between the source and the lens) and the image distance (distance between the image and the lens) for any surface termination of the PhC lens. It is observed that the excitation of surface waves does not necessarily pull the image closer to the lens. The effects of the thickness and the lateral width of the lens are also analyzed. @ 2012 Optical Society of America OCIS codes: $\quad 230.5298,240.6690,110.2960$.
\end{abstract}

\section{INTRODUCTION}

In 1968, Veselago [1] predicted that a planar slab of material with refractive index of -1 acts as a lens and focuses the electromagnetic waves from a point source. In 2000, Pendry [2] proved that the evanescent waves will be amplified inside negative index lenses and their amplitude at the image would be the same of the source. Evanescent waves, which carry subwavelength details of the source, decay and vanish in the conventional lenses, and therefore the image of conventional lenses does not include the subwavelength information of the source. In other words, in conventional lenses, only the propagating waves participate in image formation and the lens resolution is limited to $N 2$ (diffraction limit) [2,3]. On the contrary, in negative index lenses, both propagating waves and evanescent waves contribute to the image formation and the image will contain all the information of the source, and that is why these lenses are called perfect lenses. Having flat surfaces and no optical axis are other interesting properties of perfect lenses [2].

Materials with a negative index of refraction do not exist naturally, although various structures have been proposed that exhibit negative refractive index in some specific frequency regions. One of these structures is photonic crystals (PhCs) [4,5]. By proper design, various dispersion diagrams can be obtained for these structures [6]. Since the constituents of PhCs are ordinary dielectrics, their loss is low and they can be used in optical frequencies. Thus their application in negative index lenses has been investigated extensively and several approaches have been proposed [ㄱ-17].

Luo et al. [7] proposed a PhC slab lens with a square lattice, working in the first band of the PhC. This lens is inherently different from Veselago's lens in that the equifrequency contours (EFCs) are anisotropic and have a specific shape, where the EFCs of the Veselago's lens are circular and isotropic. The source should be located in the near field of this $\mathrm{PhC}$ lens, and the image is formed in the vicinity of the lens as well. Furthermore, the dominant mechanism of lensing is self-collimation rather than negative refraction [7-10]. Excitation of surface modes on lateral interfaces of the lens and the interaction between the two has been investigated in [7].

Another form of the $\mathrm{PhC}$ lens has been introduced in [11]. In this type of lens, both propagating waves and evanescent waves are transferred along the slab via canalization. Propagating waves and evanescent waves couple to the eigenmodes of the PhC and travel along the lens and eventually construct the image $[\underline{11}, \underline{12}]$. Unlike the Luo structure and Pendry lens, negative refraction and amplification of the evanescent waves does not occur in this lens (except for a very narrow spectrum of evanescent waves).

Another lensing approach in PhCs is based on isotropic EFCs [13-15]. This category of PhC slab lenses is very similar to the Pendry perfect lens in the sense that an effective phase index can be defined and is equal to $-1[4,5]$. Also the phase velocity and group velocity are antiparallel. Furthermore, the $\mathrm{PhC}$ lens roughly follows the geometric optic formula and the summation of source distance (distance between the source and the slab) and image distance (distance between the image and the slab) is nearly equal to the slab thickness $\left(d_{\mathrm{src}}+\right.$ $d_{\text {img }} \approx W_{\text {slab}}$ ). The propagating waves (far-field components) of the source will refract in the negative direction at the first interface of the slab and will cross inside the lens, forming an image within the lens. They refract negatively at the second interface as well and form another image outside of the lens.

Despite the similarities, there are differences between this PhC lens and the perfect lens of Pendry. In the case of the perfect lens, the refractive index is equal to -1 and the impedance of the lens and the impedance of the air are matched. Thus there is no reflection off the interfaces [1,2]. However, in PhCs, the effective phase index of -1 neither describes the transmissive/reflective behavior of the $\mathrm{PhC}$, nor does it mean 
a reflectionless lens $[\underline{4}, \underline{18}]$. The electromagnetic waves experience reflections at the interfaces, and therefore the transfer function of the PhC lens (in contrary to the perfect lens) is not unity for all spatial components of the source $[14,16]$; i.e., some parts of the information are lost in the image. Furthermore, the evanescent waves are reconstructed within the PhC lens only via excitation of surface modes. Also, the amount of reconstruction of evanescent waves within the $\mathrm{PhC}$ is different for various spatial frequency components, which makes the perfect reconstruction of these components impossible [3,16].

In recent years, a superlensing effect with effective phase index of -1 has been reported in several references [13-15,17]. However, a systematic study of this lens and investigation of the effects of various structural parameters on the image quality is lacking. In this paper, the impacts of the structural parameters on the properties of the image have been investigated systematically. The influences of surface termination, source distance, thickness, and lateral width of the lens are examined.

\section{THEORY AND NUMERICAL ANALYSIS}

A two-dimensional (2D) PhC structure with finite thickness and finite lateral width, surrounded by air, is simulated and analyzed. The PhC consists of a triangular array of cylindrical holes (infinitely long in the $z$ direction) with radius $r=0.4 a$ in a nonmagnetic dielectric with permittivity of $\epsilon_{r}=12.96$, where $a$ is the lattice constant of the $\mathrm{PhC}$ [13-17]. The surface termination of the structure is denoted by $\bar{C}$. When the cylinders at the lateral interfaces are perfectly shaped (full cylinders), $C$ is considered to be zero, and when the cylinders are partially cut, $C$ is considered to have a positive value. A TM (the electrical field parallel to the cylinders) monochromatic point source with normalized frequency of $f=\omega \alpha 2 \pi c$ [ $\underline{6}]$ is located on the left side of the structure at a distance $d_{\text {src }}$. An image of the source is formed on the right side of the structure at a distance $d_{\text {img }}$. A typical structure with $n_{x}=9$ and $n_{y}$ is depicted in Fig. 1 .

The photonic band structure for the $\mathrm{PhC}$ is calculated by numerically solving the Maxwell equations using the standard plane wave expansion method [6]. The band structure for the first three bands of the TM mode is depicted in Fig. 2. The EFCs of the second band of the TM mode are depicted in Fig. 3(a). It can be seen that the contours are almost circular. Thus an effective refraction index $\left|n_{\text {eff }}\right|=k c / \omega$ can be defined, where $c$ is the light velocity in vacuum and $k$ is the magnitude of the wave vector $[4,5]$. The radius of EFCs decreases as the frequency increases, and therefore group velocity vector $\left(\mathbf{v}_{g}=\nabla_{k} \omega\right)$ points inward the EFCs. Hence the effective refractive index is negative at this frequency range [4, $]$ ]. $n_{\text {eff }}$ has been calculated and plotted at different frequencies in Fig. 3(b). It can be seen that for normalized frequency of $f=0.3$ the refractive index is equal to -1 . To have a single beam propagation and avoid multirefringence in this frequency, a number of conditions should be satisfied [19],

- Only a single band solution must exist at this frequency (second band in Fig. 2).

- The band has to have a monotonic shape (Fig. 2) for the frequency region of interest.

- $\left|n_{\text {eff }}\right|<\frac{1}{2 C_{\text {str.cuf }}}$, where in this lens $f=0.3$ and $C_{\text {str,cut }}=$ 1 [19], which results in $\left|n_{\text {eff }}\right|<1.66$ [Figure 3(b)].

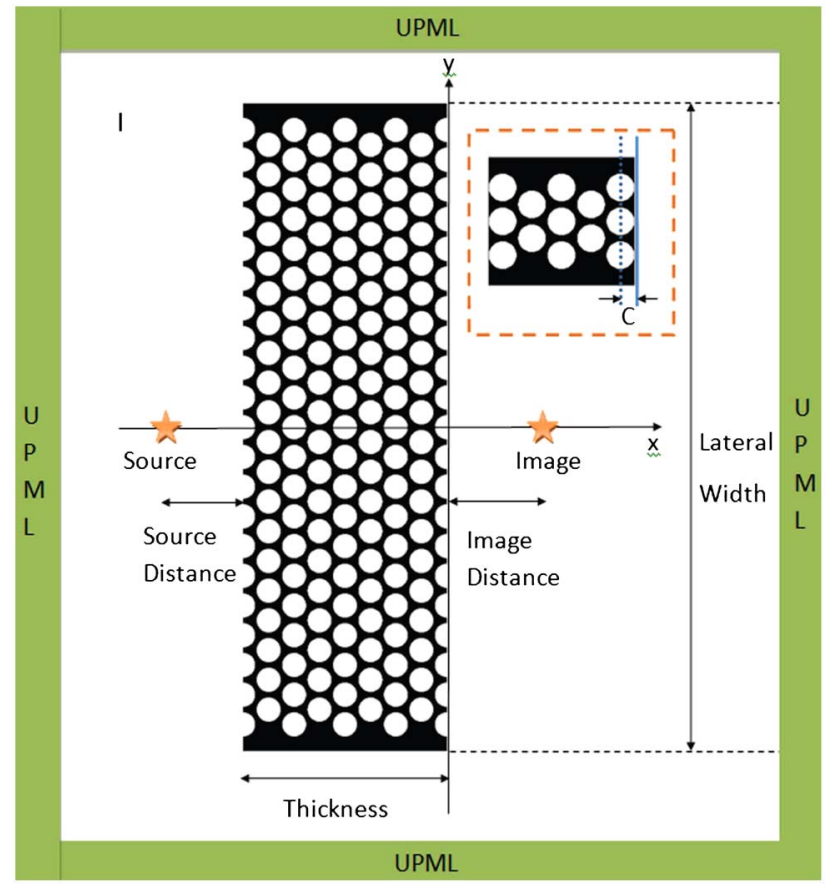

Fig. 1. (Color online) Typical structure with $r=0.4 a, C=0.4 a$, $n_{x}=9$ and $n_{y}=31$. Circles at the left interface and the right interface of the PhC structure are cut in half $(C=r=0.4 a)$. The surface termination is denoted by $C$.

Therefore, a PhC structure with these properties could work as a superlens at this frequency.

To study the lensing properties of this $\mathrm{PhC}$, a time domain simulator based on the finite-difference time-domain method has been developed [20]. The uniaxial perfectly matched layer is employed as absorbing boundary area around the structure $[20,21]$. In the next section, the effects of different physical specification of the lens on the image properties are studied.

\section{EFFECTS OF PHYSICAL SPECIFICATIONS OF THE PhC LENS ON IMAGE PROPERTIES}

\section{A. Surface Termination}

In this subsection, effects of the surface termination of the PhC structure on the lens quality and the image properties have been investigated [14,22-24]. The magnitude of the Poynting vector $|S|=\sqrt{\mathbf{S} \cdot \mathbf{a}_{x}^{2}+\mathbf{S} \cdot \mathbf{a}_{y}^{2}}$ is averaged at each

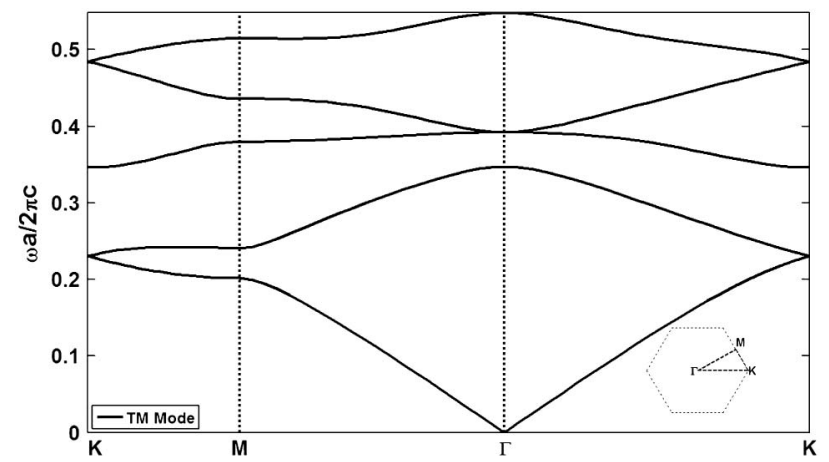

Fig. 2. Photonic band structure of TM bands of a 2D PhC composed of triangular lattice of cylindrical air holes of radius $r=0.4 a$ in dielectric with permittivity $\epsilon_{r}=12.96$. 

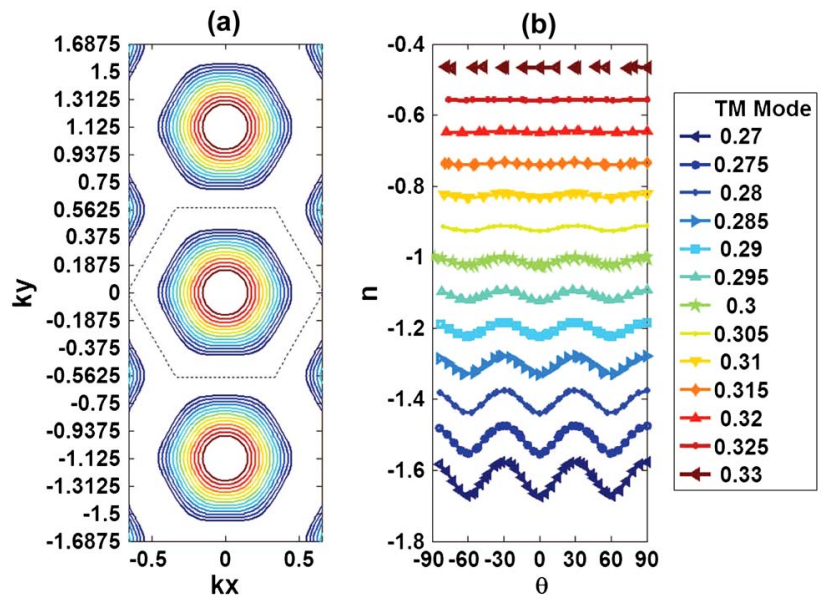

Fig. 3. (Color online) (a) EFCs for the second TM band of the PhC introduced in Fig. 2. (b) Effective phase index at each frequency versus the incident angle for (a). At $f=0.3$, the EFC is almost circular and the effective phase index is -1 .

point over a full wave period at steady state $\left(S_{\text {ave }}\right)$. The image location is defined as the position of maximum $\left|S_{\mathrm{ave}, T}\right|$ in the image plane (right side of the lens), and FWHM is measured as the full-width at half-maximum (FWHM) of the image along the $y$ direction at the image point. The FWHM, image distance, and field intensity at the image versus the surface termination are plotted in Fig. $\underline{4} . n_{x}=9, n_{y}=31, d_{\text {src }}=3 a$, and $f=0.3$ are considered for the structure. As discussed in the next section, for sources too close or too far from the lens, there are other factors that affect the image. $d_{\mathrm{src}}=3 a$ is chosen to avoid those factors as much as possible. Also for this source distance, the source is in the near field of the lens and surface states could be excited effectively.

It can clearly be seen in Fig. $\underline{4}$ that the surface termination has a significant effect on the image properties. A closer look at Fig. 4(a) reveals that for $C=0.12 a$, the FWHM has the smallest value compared to the other surface terminations. It is also observed that this FWHM $(0.4 \lambda)$ has a value that is smaller than $0.5 \lambda$, meaning that the lens is working as a superlens where the diffraction limit has been overcome to some extent. Moreover, it can be seen in Fig. 4(a) that the image intensity is the highest for this specific surface termination. Putting all this together, it can be concluded that the best image is obtained for $C=0.12 a$ in this PhC lens.

To get a further insight into the above-mentioned results, the power flux within the $\mathrm{PhC}$ is investigated. The time-averaged

(a)

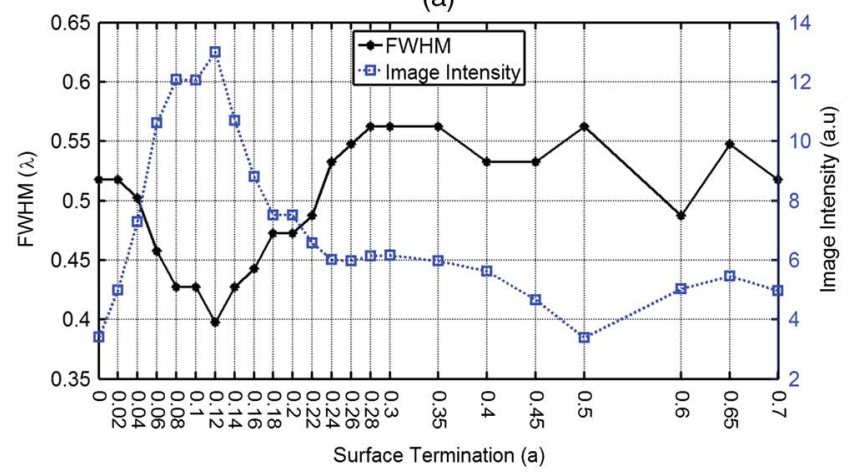

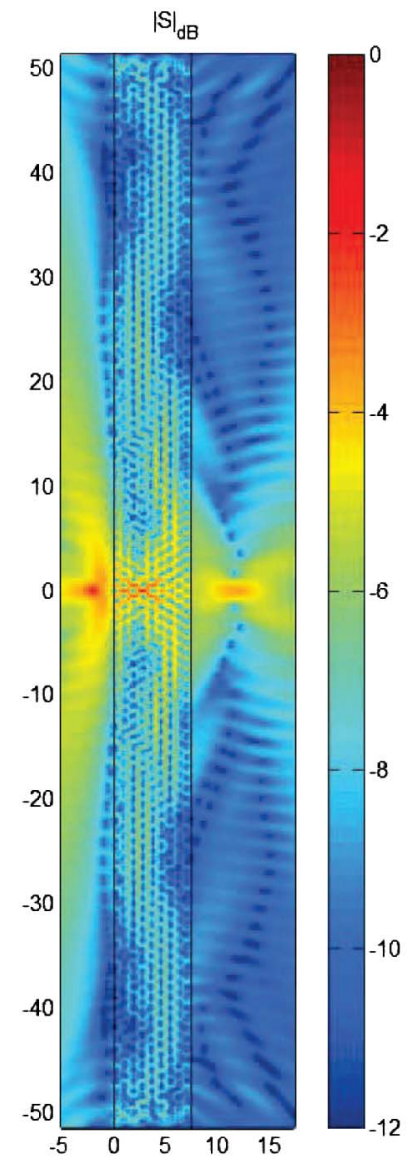

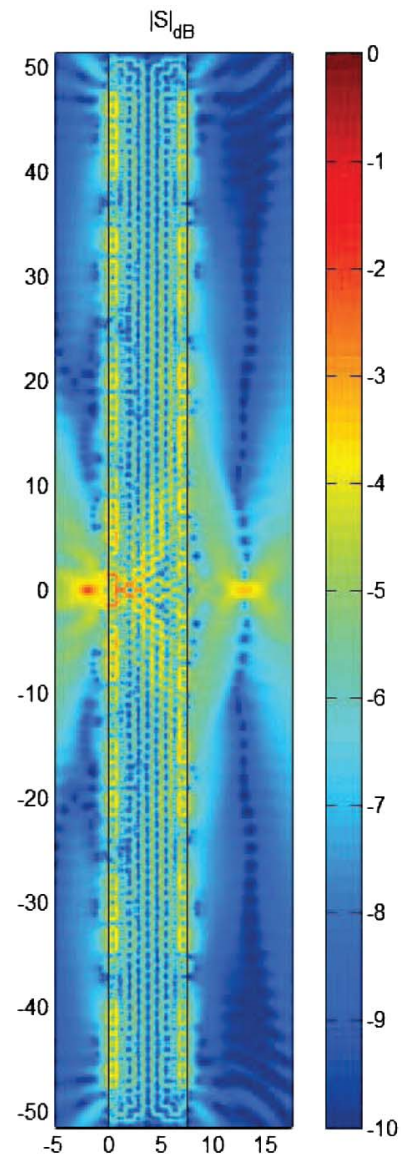

Fig. 5. (Color online) Time-averaged magnitude of Poynting vector in logarithmic scale for (a) $C=0.2 a$ and (b) $C=0.12 a$. In both figures, $n_{x}=9, n_{y}=101$, and $f=0.3$. The source is located at $y=0$ at the left side of the lens, and the image is formed at the right side of the structure. The borders of the lens are shown by black lines.

magnitude of the Poynting vector, $S_{\text {ave } T}$, is depicted in logarithmic scale in Figs. $5(\mathrm{a})$ and $5(\mathrm{~b})$ for $C=0.2 a$ and $C=0.12 a$, respectively. $n_{y}=101$ is considered for both cases. It can be seen in Fig. 5(b) that the power pattern at the interfaces and inside the PhC is notably modified compared to Fig. $5(\mathrm{a})$. A close look at the power magnitude at the interfaces of the lens in Fig. $5(\mathrm{~b})$ reveals high concentration of energy in these regions, which indicates the existence of surface waves on the interfaces of the lens [ㄴ,15]. On the other hand, by a closer look at Fig. 4(b) it can be observed that the excitation of surface

(b)

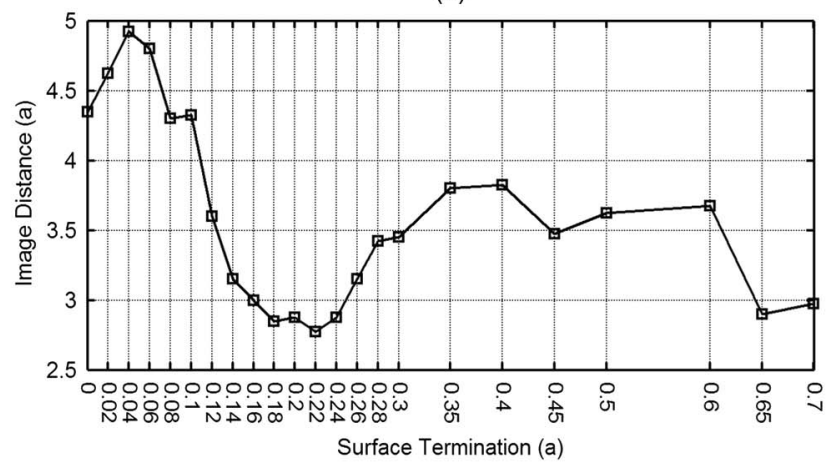

Fig. 4. (Color online) FWHM and the (a) intensity of the image and (b) image distance, for different surface terminations. In both figures, $n_{x}=9$, $n_{y}=31$, and the source distance is $3 a$. The smallest FWHM and the highest image intensity are obtained for $C=0.12 a$. 
waves for $C=0.12 a$ has not pulled the image closer to the second interface of the lens and in general, a very complicated behavior exists in the image distance which cannot be explained by the surface wave excitation.

\section{B. Source Distance}

In this subsection, the effects of the distance between the source and the lens on the image properties are examined. The lens is expected to obey the rules of ray optics, meaning that the summation of the source distance and the image distance to be equal to the lens thickness [2]]:

$$
d_{\mathrm{src}}+d_{\mathrm{img}}=W_{\mathrm{slab}}
$$

where for this PhC structure, $W_{\text {slab }}=\left(n_{x}-1\right) \frac{\sqrt{3}}{2}+2 r-2 C$. Two surface terminations of $C=0.1 a$ and $C=0.2 a$ are examined, where $C=0.1 a$ is very close to the optimum case $(C=0.12 a)$ and $C=0.2 a$ is an arbitrary termination. The image distance versus the source distance for these surface terminations $(C=0.1 a$ and $C=0.2 a)$ is plotted in Fig. 6 . Lenses with $n_{x}=9$ and $n_{y}=31$ are used, and the normalized source frequency is set to $f=0.3$. It is observed that the image distance depends on the surface termination. The thickness of the structures with $C=0.1 a$ and $C=0.2 a$ are $7.53 a$ and $7.33 a$, respectively. The difference between the two thicknesses is equal to $0.2 a$. Thus, it is expected from (1) that for a specific source distance, the difference between the image distances of these lenses is equal to $0.2 a$, too. But as can be seen in Fig. 6, the image distances are more than $1 a$ apart for each source distance, which implies that Eq. (1) does not hold exactly in PhC lenses.

On the other hand, when the source distance is increased (decreased), the image is moved closer to (farther from) the lens, which is consistent with the rules of geometric optics [2] and very interestingly, there exists a linear dependence of the image distance to the source distance [25]. It is concluded that in the PhC lenses, Eq. (1) can be rewritten as

$$
d_{\mathrm{src}}+d_{\mathrm{img}}=\alpha_{c} W_{\mathrm{slab}},
$$

where $\alpha_{c}$ is a constant value that depends on the surface termination and should be calculated for each value of $C$ separately. The same results are observed for other surface terminations. $\alpha_{c}=0.84$ and $\alpha_{c}=0.66$ are obtained for $C=0.1 a$ and $C=0.2 a$, respectively, where Fig. $4(\mathrm{~b})$ can be used to calculate the value of $\alpha_{c}$ for other surface terminations.

Rules of ray optics impose a limitation on the source distance in a negative index lens where an image is formed only when the source distance is smaller than the lens thickness $[\underline{2}, \underline{3}]$. In a PhC lens, there are three source distance criteria, as follows:

1. For source distances smaller than a specific value $d_{\text {lin }}$, the image distance has a linear dependence on the source distance and equation (2) holds. As can be seen in Fig. $\underline{6}, d_{\text {lin }}$ is almost equal to $6.5 a$ and $5 a$ for $C=0.1 a$ and $C=0.2 a$, respectively. Note that observing a linear dependence of the image distance to the source distance in a PhC lens is remarkable and of the great importance in constructing $3 \mathrm{D}$ images from objects in the imaging applications.

2. For source distances between $d_{\text {lin }}$ and $d_{\max }$, the image is formed in the vicinity of the lens and its distance does not

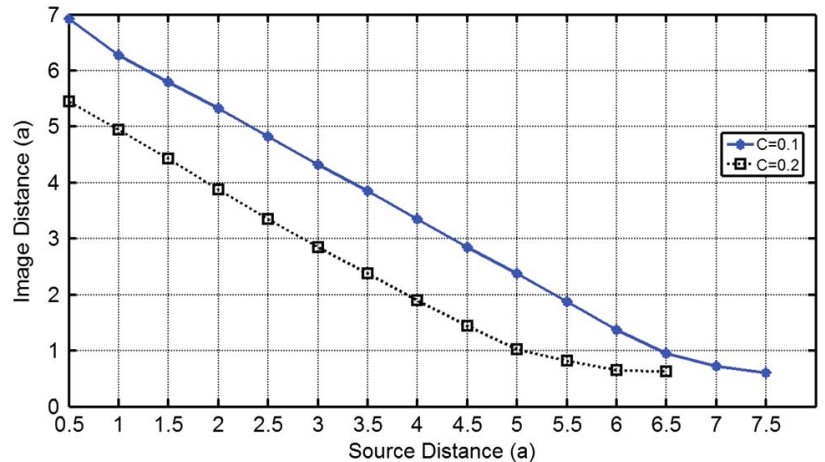

Fig. 6. (Color online) Image distance versus the source distance for $C=0.1 a$ and $C=0.2 a$. In both figures, $n_{x}=9, n_{y}=31$, and $f=0.3$. The image vanishes for source distances larger than $6.5 a$ and $7.5 a$ for $C=0.2 a$ and $C=0.1 a$, respectively.

have the linear dependency on the source distance [17]. $d_{\max }$ is defined as the upper limit of the source distance where an image would form in a PhC lens. It changes based on the lens structural parameters and should be calculated separately for each structure. In Fig. $\underline{6}, d_{\max }$ is almost equal to $7.5 a$ and $6.5 a$ for lenses of $C=0.1 a$ and $C=0.2 a$, respectively.

3. For source distances larger than $d_{\max }$, superfocusing does not occur anymore. The spatial frequencies of the source does not meet inside the lens, thus no image, neither inside nor outside of the lens, would be formed.

FWHM and intensity of the image versus the source distance are shown in Fig. 7 for two cases of $C=0.1 a$ and $C=0.2 a$. It can be seen that in both cases, for source distances between $d_{\text {lin }}$ and $d_{\max }$, the FWHM is highly increased. There are two mechanisms involved in this phenomenon. First, when the image has a large distance from the lens, the evanescent waves vanish before reaching the image and therefore the resolution diminishes. The second reason is the limited lateral width of the PhC lens (in this case $n_{y}=31 a$ ). In fact when the source is moved further from the lens, the incident wave with a large incident angle (with respect to the normal to the structure) does not hit the lens and therefore does not participate in imaging. As evidence, the lens with $n_{y}=51, C=0.2 a$, and the source distance equal to $6 a$ has a FWHM $=0.47 \lambda$ and image intensity of 7.3 , which shows a smaller FWHM and a higher intensity than those of the $n_{y}=$ 31 (FWHM $=0.49 \lambda$ and image intensity of 6.8). A similar effect has been observed for the anisotropic perfect lens, where the degradation of the image for larger distances has been attributed to the finite lateral size of the lens [25].

Another observable fact in Fig. 7 is the increase of FWHM for sources very close to the lens. When the source is very close to the lens, the image is formed very far from the lens and therefore the effect of the evanescent waves on the image is vanished. Moreover, the source is in the near field of the lens and the scattering effects are stronger. These two factors lead to an image with a larger FWHM and lower intensity if the source is at a small distance from the lens.

\section{Lateral Width of the PhC Lens}

In this subsection, the effects of the lateral width of the lens on the image properties are investigated by changing $n_{y}$. The first guess is that increasing the lateral width would improve the lens quality. This is because a greater fraction of the source 
(a)

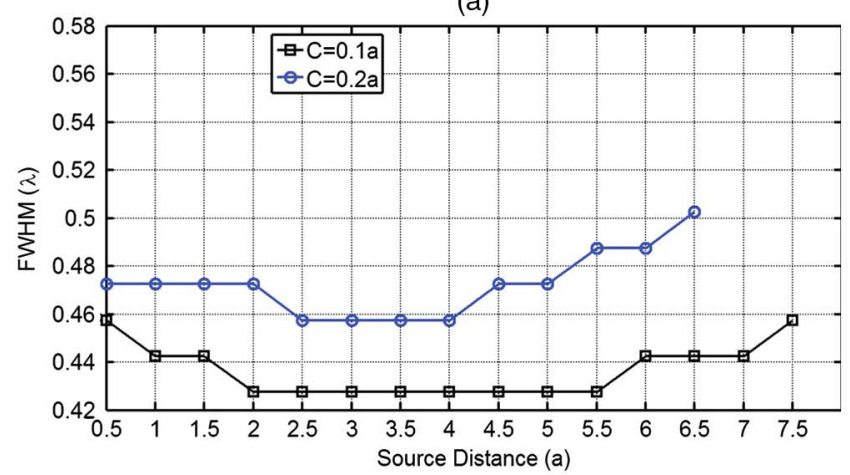

(b)

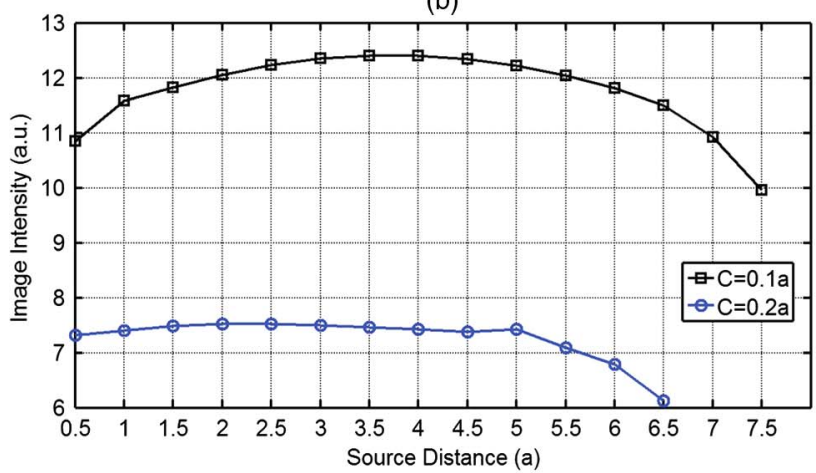

Fig. 7. (Color online) (a) FWHM and (b) image intensity versus the source distance for two different surface terminations. The image quality decreases as the source moves too close to or too far from the lens.

power would hit the lens and lower fraction of the source information is lost. In other words, the numerical aperture of the lens increases.

To confirm this, structures with $n_{y}=11, \ldots, 41,51$, $85,101,151$ are simulated and the image properties for each structure at frequency $f=0.3$ are calculated. The surface termination is considered to be $C=0.2 a$ and the source distance is $2 a$. FWHM and image intensity are represented in Fig. $\underline{8}$.

It can be seen that when two specific structures are compared, the image quality is not necessarily better for the larger structure. For example, the FWHM is bigger for $n_{y}=32$ than that of $n_{y}=30$, where it is expected otherwise. This random behavior is also obvious in the image intensity. But in general, it can be seen that the image quality improves when the lateral

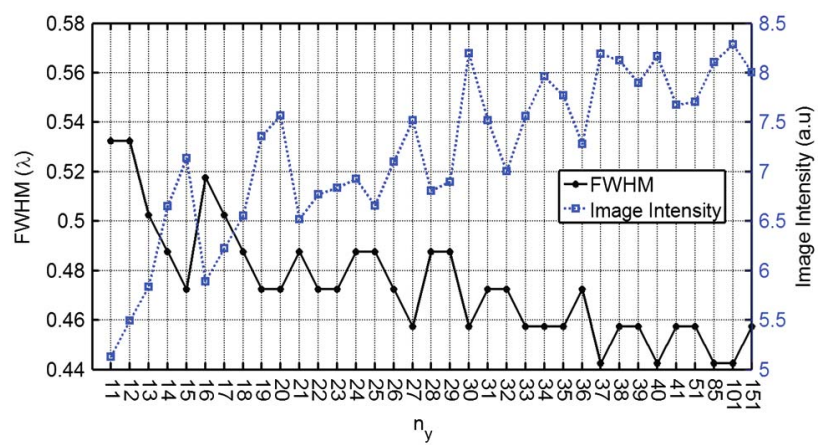

Fig. 8. (Color online) FWHM and the image intensity versus $n_{y}$. The FWHM has a negative average slope and the image intensity has a positive average slope. width is increased, and the image has lower FWHM and a higher image intensity for larger structures. It can also be seen that the FWHM has a negative average slope and the image intensity has a positive average slope. Based on this analysis, it is concluded that the lateral width of the $\mathrm{PhC}$ lens is an influential parameter on the image quality and increasing the lateral width would generally improve the performance of the lens.

However, some other mechanisms are also involved in this process, which have the dominant effect on the image properties for some structures. One of these mechanisms is the edge effects of the PhC structure and the interactions and cancellations of the fields at the edges of the structure. Investigating the power flux in upper and lower regions of the lens reveals a complicated behavior for different lateral widths.

\section{Thickness of the Lens}

In this subsection, the effect of lens thickness on the image quality is investigated by changing $n_{x}$. For this purpose, structures with $n_{y}=31$, and $n_{x}=6,12,16,32$ are considered. The electric field pattern $|\mathbf{E}|$ of the right side of the lens (image side) in an arbitrary time (after reaching steady state) is plotted in Fig. 9. The simulation has been performed for long enough time for the image to form. It can be seen that as the thickness of the structure increases, the distortion in the pattern of image increases and consequently the image quality deteriorates. Increasing the thickness, keeping in mind that $d_{\text {src }}+d_{\text {img }}=\alpha_{c} W_{\text {slab }}$, means that the image will be formed in a longer distance from the structure. This means that, considering the limited lateral width of the lens, the image will be fed from the waves with smaller incident angles (with respect
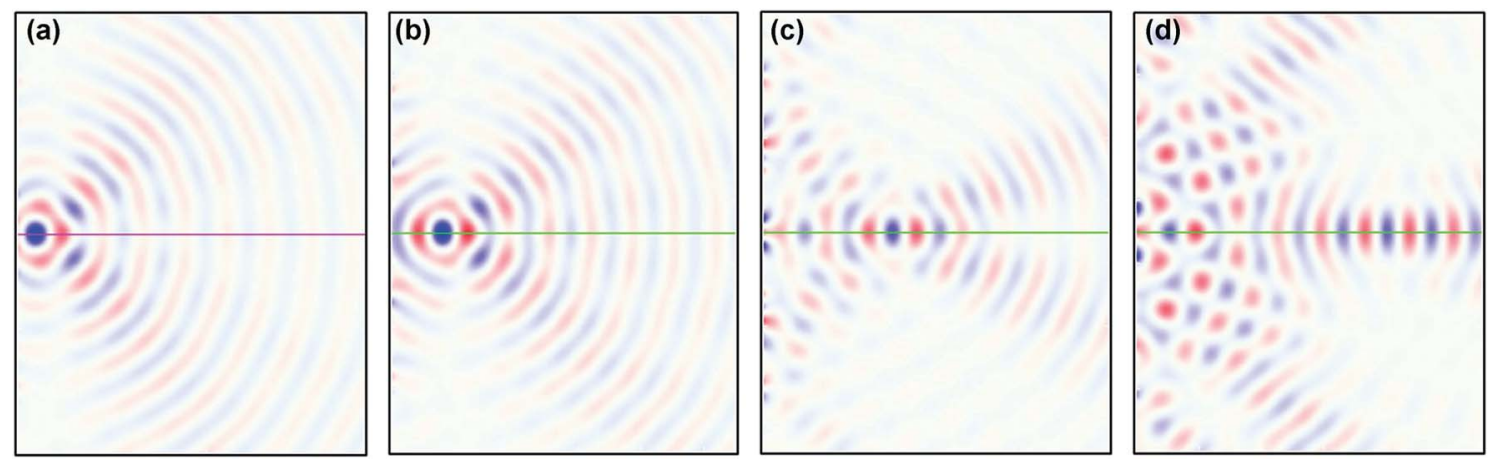

Fig. 9. (Color online) Image side of the lens for different lens thicknesses. $n_{x}=6,12,16,32$ from left to right. As the thickness of the structure increases, the distortion in the pattern of image increases. $C=0.2 a$ is used in all cases. 
to the normal to the lens). In other word, the numerical aperture of the image will be smaller. Furthermore, the effects of the evanescent waves on the image will vanish when the image is too far from the lens. It can be concluded that increasing the thickness generally deteriorates the image.

\section{CONCLUSION}

In summary, the effects of the surface termination is studied by investigating the power flux within the $\mathrm{PhC}$ lens. It is observed that for certain surface terminations, surface waves are excited at the interfaces of the lens, which notably improve the image quality. Although the excitation of surface waves does not necessarily pull the image closer to the lens and a complicated relationship exists between the image distance and the surface termination. Also by studying the source distance and the image distance, it is observed that the $\mathrm{PhC}$ lens obeys the rules of geometric optics to some extent and a linear relation between the source distance and the image distance has been obtained. It is shown that increasing the $\mathrm{PhC}$ lateral width would generally enhance the image quality by increasing the lens optical aperture. On the contrary, increasing the thickness might deteriorate the image because of the enhanced reflection and scattering effects.

\section{REFERENCES}

1. V. Veselago, "The electrodynamics of substances with simultaneously negative values of $\epsilon$ and $\mu$," Sov. Phys. Usp. 10, 509-514 (1968).

2. J. B. Pendry, "Negative refraction makes a perfect lens," Phys. Rev. Lett. 85, 3966-3969 (2000).

3. S. Anantha Ramakrishna, "Physics of negative refractive index materials," Rep. Prog. Phys. 68, 449-521 (2005).

4. S. Foteinopoulou and C. M. Soukoulis, "Negative refraction and left-handed behavior in two-dimensional photonic crystals," Phys. Rev. B 67, 235107 (2003).

5. M. Notomi, "Theory of light propagation in strongly modulated photonic crystals: Refraction like behavior in the vicinity of the photonic band gap," Phys. Rev. B 62, 10696-10705 (2000).

6. J. D. Joannopoulos, R. D. Meade, and J. N. Winn, Photonic Crystals: Molding the Flow of Light (Princeton University, 1995).

7. C. Luo, S. G. Johnson, and J. D. Joannopoulos, "Subwavelength imaging in photonic crystals," Phys. Rev. B 68, 045115 (2003).

8. E. Cubukcu, K. Aydin, and E. Ozbay, "Subwavelength resolution in a two-dimensional photonic-crystal-based superlens," Phys. Rev. Lett. 91, 207401 (2003).
9. Z.-Y. Li and L.-L. Lin, "Evaluation of lensing in photonic crystal slabs exhibiting negative refraction,” Phys. Rev. B 68, 245110 (2003).

10. C. Luo, S. G. Johnson, and J. D. Joannopoulos, “All-angle negative refraction without negative effective index," Phys. Rev. B 65, 201104 (2002).

11. P. A. Belov and C. R. Simovski, "Canalization of subwavelength images by electromagnetic crystals,” Phys. Rev. B 71, 193105 (2005).

12. Y. Liu, G. Bartal, and X. Zhang, "All-angle negative refraction and imaging in a bulk medium made of metallic nanowires in the visible region,” Opt. Express 15439-15448 (2008).

13. X. Wang, Z. Ren, and K. Kempa, "Unrestricted superlensing in a triangular two dimensional photonic crystal," Opt. Express 12, 2919-2924 (2004).

14. R. Moussa, S. Foteinopoulou, Lei Zhang, G. Tuttle, K. Guven, E. Ozbay, and C. M. Soukoulis, "Negative refraction and superlens behavior in a two-dimensional photonic crystal," Phys. Rev. B 71, 085106 (2005)

15. X. Wang and K. Kempa, "Effects of disorder on subwavelength lensing in two-dimensional photonic crystal slabs," Phys. Rev. B 71, 085101 (2005).

16. S. Xiao, M. Qiu, Z. Ruan, and S. He, "Influence of the surface termination to the point imaging by a photonic crystal slab with negative refraction,” Appl. Phys. Lett. 85, 4269-4271 (2004).

17. G. Sun, A. S. Jugessur, and A. G. Kirk, "Imaging properties of dielectric photonic crystal slabs for large object distances," Opt. Express 14, 6755-6765 (2006).

18. S. Foteinopoulou, "Photonic crystals as metamaterials," Phys. B, doi:10.1016/j.physb.2012.01.092 (to be published).

19. S. Foteinopoulou and C. M. Soukoulis, "Electromagnetic wave propagation in two-dimensional photonic crystals: a study of anomalous refractive effects," Phys. Rev. B 72, 165112 (2005)

20. A. Taflove, Computational Electrodynamics: The Finite Difference Time-Domain Method (Artech House, 1995).

21. J. Berenger, "A perfectly matched layer for the absorption of electromagnetic waves,” J. Comput. Phys. 114, 185-200 (1994).

22. N. Fang, Z. Liu, T.-J. Yen, and X. Zhang, "Experimental study of transmission enhancement of evanescent waves through silver films assisted by surface plasmon excitation," Appl. Phys. A 80, 1315-1325 (2005).

23. E. Moreno, F. J. Garcia-Vidal, and L. Martin-Moreno, "Enhanced transmission and beaming of light via photonic crystal surface modes," Phys. Rev. B 69, 121402 (2004).

24. R. Moussa, Th. Koschny, and C. M. Soukoulis, "Excitation of surface waves in a photonic crystal with negative refraction: the role of surface termination,” Phys. Rev. B 74, 115111 (2006).

25. N. Shen, S. Foteinopoulou, M. Kafesaki, T. Koschny, E. Ozbay, E. Economou, and M. Soukoulis, "Compact planar far-field superlens based on anisotropic left-handed metamaterials," Phys. Rev. B 80, 115123 (2009). 